\title{
L'huile de pépins de raisin en France et dans le monde
}

\author{
Jean-Claude Pierron* \\ Société Provence Huiles, 25 ZI deuxième avenue, 13127 Vitrolles, France
}

Reçu le 25 avril 2017 - Accepté le 13 juin 2017

\begin{abstract}
Résumé - Avec une production mondiale de l'ordre de 35000 tonnes, dont 10000 tonnes émanant des usines françaises, l'huile de pépins de raisin est un produit de niche. S'il affiche aujourd'hui des prix plus de deux fois supérieurs aux huiles de gros volumes comme le tournesol, ce produit était encore à la recherche de débouchés il y a encore peu. En effet, le pépin de raisin, et plus généralement le marc, était encore dans les années 1970 un coproduit de l'industrie vinicole auquel il fallait trouver un débouché, suite à l'interdiction de leur épandage en agriculture. Aujourd'hui, à l'issue d'un process au rendement relativement faible, 70000 tonnes de pépins de raisin issus de la vinification française permettent d'extraire 11 à 12000 tonnes d'huile brute, qui vont générer 10000 tonnes environ d'huile raffinée. Profitant d'une bonne image nutritionnelle et de qualités technologiques (fluidité), l'huile de pépins de raisin a su conquérir l'export, et notamment les États-Unis et la Corée du Sud.
\end{abstract}

Mots clés : huile / pépins de raisin / marc

\begin{abstract}
Grape seed oil in France and in the world. With a total global annual production of approximately 35000 tons, of which 10000 tons are from French factories, grape seed oil is a niche product. Though its price is now over twice that of high-volume oils like sunflower, until only recently this product was still in search of outlets. Indeed, grape seed, and marc more generally, was still in the 1970s no more than a byproduct of the wine industry for which, since its dispersion across agricultural land had been prohibited, it was necessary to identify an end-use. Today, via a relatively low-yielding process, 70000 tons of grape seeds derived from French winemaking produce annually between 11 and 12000 tons of crude oil, which results in approximately 10000 tons of refined oil. Drawing on its positive nutritional image and technological qualities (fluidity), grape seed oil has gained export markets, notably in the United States and South Korea.
\end{abstract}

Keywords: grape seed oil / marc

\section{Un coproduit à haute valeur ajoutée}

\subsection{Les marcs}

Les pépins de raisin dont est issue l'huile éponyme, sont des coproduits de l'industrie vinicole. En effet, après vinification du raisin, se trouvent, en fond de cuve, les marcs. Ces derniers sont extraits puis livrés aux distilleries qui sont surtout installées dans le Sud de la France. Les marcs reçus par les distilleries contiennent en moyenne de l'ordre de $15 \%$ de pépins, $30 \%$ de pulpes et rafles, et $55 \%$ d'eau. La collecte provient du grand Sud de la France : région Cognac et Bordeaux en premier, puis région Midi-Pyrénées et Provence-Alpes Côtes d'Azur (PACA), Vallée du Rhône en partie.

\footnotetext{
*Auteur correspondant : jc.pierron56@gmail.com
}

\subsection{Un coproduit progressivement valorisé}

L'industrie de valorisation des coproduits de l'industrie vinicole est née de l'obligation de ne plus destiner les marcs à l'épandage agricole dans les années 1970-1980. Cette valorisation reposait principalement sur deux produits extraits de ces marcs: l'alcool éthylique qui était alors contingenté avec une valeur marchande confortable et fixée par l'état; et le tartrate également vendu à un bon niveau de prix, car encore peu concurrencé par le tartrate de synthèse chinois. Quant aux pépins de raisin et à la pulpe, leurs ventes ne représentaient qu'une maigre part du chiffre d'affaires de l'activité.

Aujourd'hui, la tendance s'est totalement inversée : après la mise en vente de l'alcool sur le marché libre et la concurrence féroce sur le tartrate, la valeur ajoutée de la valorisation des marcs de vinification repose désormais sur les revenus générés par les huiles de pépins et par la pulpe, notamment grâce au succès commercial de la vente des huiles de pépins. 
En France, la valorisation des pépins de raisin était réalisée au sein de l'actuelle usine de Béziers (34) et dans une usine située à Marseille (13) fermée en 2000. Ces usines avaient dans un premier temps pour mission de transformer les pépins de raisin livrés par les distilleries en huile brute puis en huile raffinée, ainsi que la mise en bouteilles pour la mise en vente dans les caves coopératives des adhérents des distilleries et ce, en parallèle, avec les vins de la cave. Néanmoins, la demande demeurant très inférieure à l'offre, d'autres applications ont été développées : l'utilisation de l'huile de pépins de raisin comme composant de la fabrication de peintures lui a par exemple offert un nouveau débouché.

La commercialisation de cette huile en grande distribution a été très progressive; les ventes ont surtout été dopées par les qualités vantées de la haute teneur en oméga- 6 de cette huile.

\section{Les étapes de production de l'huile}

\subsection{Quatre produits issus des marcs}

Les marcs, après réception dans les distilleries, sont entassés dans des fosses puis recouverts d'une bâche de couleur noire afin de les protéger de la lumière et de l'oxygène de l'air et ainsi de minimiser au maximum leur transformation au cours du temps.

En effet, la production des marcs, et donc leur livraison, s'étale sur une courte période de temps alors que les distilleries vont traiter ces coproduits durant les 6 à 10 mois suivant leur réception.

Les marcs traités en distillerie vont produire quatre grands produits :

- l'alcool éthylique;

- le tartrate;

- le pépin de raisin;

- la pulpe.

Certaines grandes distilleries produisent également, à partir des marcs, des colorants alimentaires, des polyphénols, $\mathrm{du}$ fructose, des oligomères pro cyanidoliques (OPC).

Après tous les traitements, les coproduits de la distillerie vont être séchés, criblés et séparés. Parmi ceux-ci vont se trouver les pépins de raisin ainsi que les pulpes et rafles destinées à l'alimentation animale.

\subsection{Du pépin de raisin à l'huile}

La transformation des pépins de raisin en huile se déroule en quatre étapes :

- écrasement des pépins pour les transformer en une sorte de farine ;

- agglomération de cette farine en petits bouchons ou pellets;

- arrosage d'un lit de pellets à l'aide d'un solvant qui aura pour but d'extraire l'huile;

- séparation de l'huile du solvant par distillation.

L'huile ainsi produite porte le qualificatif d'huile brute; elle n'est pas consommable telle quelle.

Le coproduit ultime des pépins de raisin, appelé tourteau, est essentiellement constitué de fibres. Il est donc principalement utilisé en énergie thermique comme combustible. L'huile brute quant à elle est transportée dans une usine située à Vitrolles où l'on va procéder à son raffinage.
Le raffinage de l'huile brute se déroule en trois étapes successives :

- la neutralisation associée au décirage de l'huile: cette première étape consiste à éliminer les acides gras libres contenus dans l'huile brute ainsi que les acides gras à point de fusion élevé ou cires qui confèrent à l'huile un voile, voire un trouble à basse et ambiante température;

- la décoloration de l'huile : cette deuxième étape élimine la couleur plutôt foncée, voire noire, de l'huile brute;

- la désodorisation de l'huile : cette dernière étape élimine le goût désagréable de l'huile brute.

Le process de raffinage est un procédé physico-chimique.

Les difficultés majeures du raffinage de l'huile de pépins de raison concernent le décirage: en effet, les cires contenues dans l'huile brute sont longues à cristalliser et à s'agglomérer, donc à éliminer. Seconde difficulté technique : l'acidité élevée des huiles brutes produites. L'acidité oléique de l'huile brute peut en effet être très basse en début de campagne, pour évoluer très vite et fortement à la hausse.

Le rendement moyen de raffinage est modeste, de l'ordre de 85 à $90 \%$, ce qui se traduit par la production de $870 \mathrm{~kg}$ d'huile de pépins de raisin raffinée pour $1000 \mathrm{~kg}$ mis en œuvre. Rappelons que sur les huiles de table classiques, le rendement moyen est de $97 \%$ environ, soit $100 \mathrm{~kg}$ de plus par tonne d'huile mises en œuvre.

\subsection{Le rendement}

Actuellement, en France, les pépins de raisin sont livrés à Béziers (34), pour extraction de l'huile contenue dans les pépins. Cette usine est la seule en France à posséder la capacité de triturer 80 à 100000 tonnes de pépins de raisin par an.

La quantité de pépins de raisin reçue à l'usine dépend de la quantité de raisin mis en vinification, et donc de la récolte de raisin. En règle générale, l'usine d'extraction de Béziers reçoit et traite environ 70000 tonnes par an, alors que le potentiel français est de 100000 tonnes (les 30000 tonnes restantes ne sont pas traitées car logistiquement situées trop loin).

Le succès commercial de l'huile de pépins de raisin durant ces dernières années a fait considérablement évoluer son prix, entraînant dans le même mouvement haussier la valeur des pépins de raisin et donc la quantité de pépins produits par les distilleries. Ainsi, la quantité de pépins reçus à Béziers a doublé en 15 ans.

La quantité d'huile contenue dans les pépins de raisin est faible, de l'ordre de 15 à $18 \%$ en France. Elle peut être beaucoup plus basse dans d'autres pays comme l'Espagne (10 à $13 \%$ ).

Ainsi, il est nécessaire de triturer de l'ordre de $7 \mathrm{~kg}$ de pépins de raisin pour obtenir 1 litre d'huile. Ce ratio explique que la collecte des pépins de raisin sur Béziers se limite au grand Sud de la France: en effet, le transport a un impact d'un facteur 7 sur le prix de l'huile (puisqu'il faut $7 \mathrm{~kg}$ de pépins pour produire un seul $\mathrm{kg}$ d'huile). Les 30000 tonnes de marcs les plus éloignés de l'usine de Bézier ne sont donc pas traités, faute de rentabilité.

Compte tenu de ce rendement de 15 à $18 \%$ et du traitement de quelque $70000 \mathrm{t}$ de pépins de raisin par an, la production annuelle d'huile brute de pépins de raisin s'élève 11 à 12000 tonnes. Du fait d'un rendement de 85 à $90 \%$ entre les huiles brutes et raffinées de pépins de raisin, les huiles brutes produites françaises vont générer 10000 tonnes environ d'huile raffinée. 
Tableau 1.

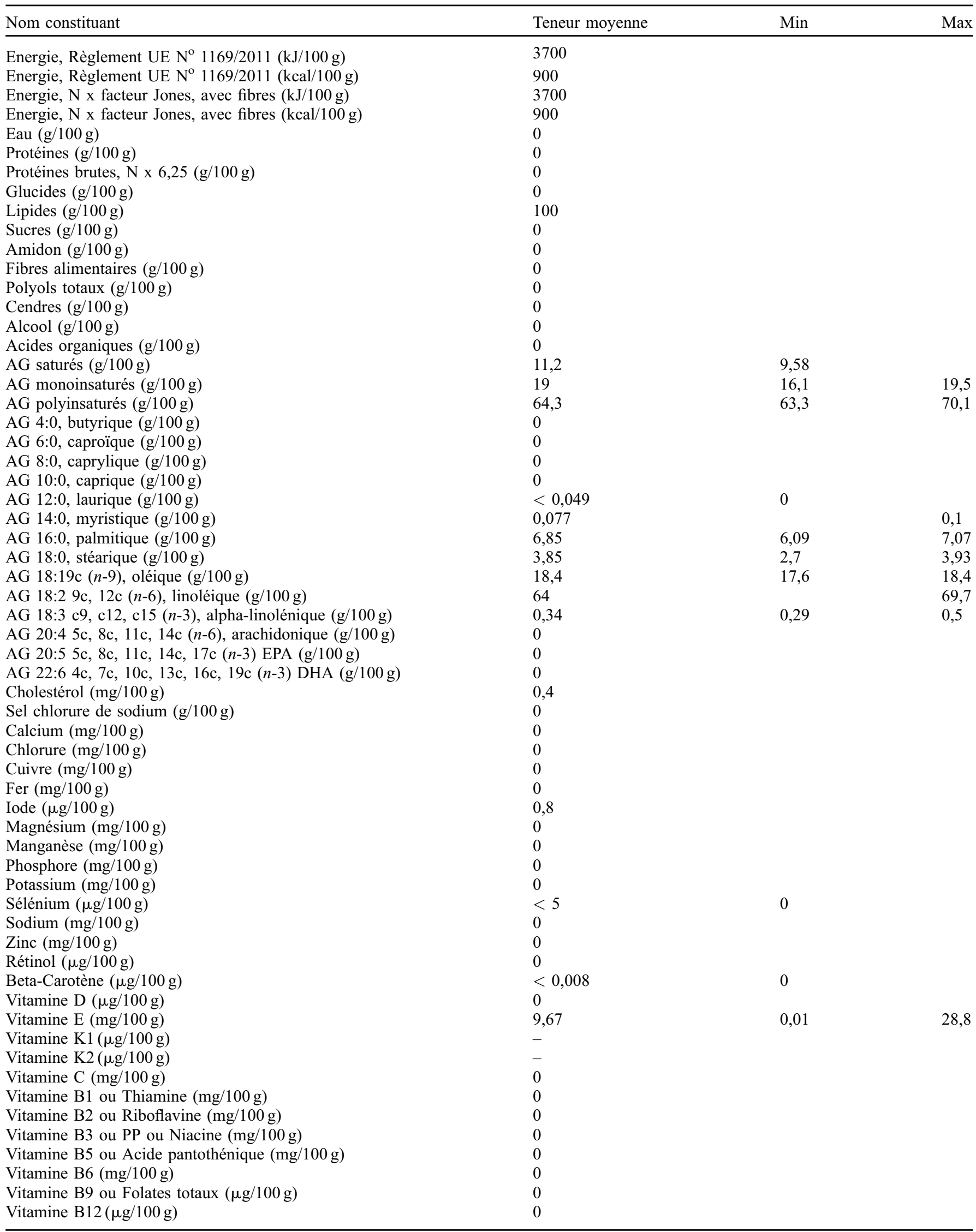


Tableau 2. Composition en acides gras de l'huile de pépin de raisin déterminée par chromatographie liquide (exprimée en pourcentage d'acides gras totaux) selon le Codex Alimentarius.

\begin{tabular}{ll}
\hline Acides gras & Huile de pépins de raisin \\
\hline C6:0 & ND \\
C8:0 $10: 0$ & ND \\
C12:0 & ND \\
C14:0 & ND \\
C16:0 $16: 1$ & ND-0,3 \\
C17:0 & $5,5-11,0$ \\
C17:1 $18: 0$ & ND-1,2 \\
C18:1 & ND-0,2 \\
C18:2 & ND-0,1 \\
C18:3 & $3,0-6,5$ \\
C20:0 $20: 1$ & $12,0-28,0$ \\
C20:2 & $58,0-78,0$ \\
C22:0 & ND-1,0 \\
C22:1 $22: 2$ & ND-1,0 \\
C24:0 & ND-0,3 \\
C24:1 & ND \\
\hline
\end{tabular}

Source: FAO/WHO, 2001.

\section{Le marché de l'huile de pépins de raisin}

\subsection{Richesse en oméga- 6 et fluidité}

Les principales qualités reconnues de l'huile de pépins de raisin par les consommateurs sont le pourcentage élevé en acide linoléique (acide gras de la famille des oméga-6) d'en moyenne $64,7 \mathrm{~g} / 100 \mathrm{~g}$ d'huile et pouvant atteindre jusqu'à 69,7 g/100 g, cf. Tableau 1 (Anses, 2016).

La grande fluidité de l'huile de pépin de raisin confère aux aliments frits à partir de cette huile un aspect qualifié de «moins gras ».

\subsection{Marché national et international}

Les clients des usines d'extraction de l'huile de pépins de raisin sont des industriels qui conditionnent principalement l'huile raffinée en bouteilles, soit tel quel, soit en assemblage avec d'autres huiles végétales. L'huile ainsi conditionnée est commercialisée principalement par la grande distribution.

Le marché annuel mondial est estimé à 35000 tonnes d'où le qualificatif de marché de niche.

Les clients sont désormais internationaux: l'exportation représente aujourd'hui $75 \%$ des volumes produits en France, alors qu'il y a une quinzaine d'années seulement, la France et l'Union européenne représentaient plus de $60 \%$ de la consommation, sur un marché quantitatif certes moindre.

Les volumes d'huile de pépins de raisin produits en Europe sont exportés vers la Corée du Sud pour 30\%, les États-Unis pour $35 \%$ et le reste du monde pour $10 \%$. À noter que ces $10 \%$ ont principalement pour destination l'Asie puisque le
Tableau 3. Spécifications de FEDIOL pour l'huile de pépins de raisin (brute et raffinée).

Composition des acides gras (exprimée en pourcentage d'acides gras totaux)

$\begin{array}{ll}\text { C6:0 } & \text { Max. } 0,05 \\ \text { C8:0 } & \text { Max. } 0,05 \\ \text { C10:0 } & \text { Max. } 0,05 \\ \text { C12:0 } & \text { Max. } 0,05 \\ \text { C14:0 } & \text { Max. } 0,3 \\ \text { C16:0 } & 5,5-11,0 \\ \text { C16:1 } & \text { Max. } 1,2 \\ \text { C17:0 } & \text { Max. } 0,2 \\ \text { C17:1 } & \text { Max. } 0,1 \\ \text { C18:0 } & 3,0-6,5 \\ \text { C18:1 } & 12,0-28,0 \\ \text { C18:2 } & 65,0-78,0 \\ \text { C18:3 } & \text { Max. } 1,0 \\ \text { C20:0 } & \text { Max. } 1,0 \\ \text { C20:1 } & \text { Max. } 0,3 \\ \text { C22:0 } & \text { Max. } 0,2 \\ \text { C22:1 } & \text { Max. } 0,3 \\ \text { C24:0 } & \text { Max. } 0,4 \\ \text { Composition de stérols (exprimée en pourcentage de stérols } \\ \text { totaux) } \\ \text { Cholestérol } \\ \text { Brassicastérol } \\ \text { Campestérol } \\ \text { Stigmastérol } \\ \text { Beta-sitostérol } 0,5 \\ \text { Delta-5-avenastérol } \\ \text { Delta-7-stigmasténol } \\ \text { Delta-7-avenastérol } \\ \text { Erythrotiol + Uvaol } \\ \text { Autres } & \text { Max. } 0,2 \\ \text { Stérols totaux (mg/kg) pour l'huile brute } & 7,5-14,0 \\ & 7,5-12,0 \\ 64,0-75,0 \\ 1,0-3,5 \\ 0,5-3,5 \\ 0,5-1,5 \\ \text { Min. 3,5 } \\ \text { Max. 5,1 } \\ 2000-7000\end{array}$

Source: FEDIOL, 2016.

Japon pèse $3 \%$, Taiwan $2 \%$, auxquels s'ajoute la Malaisie. Enfin, le Chili importe de l'ordre de $3 \%$ des volumes produits.

Aux États-Unis, l'importante production vinicole, et donc de marc, génère un immense potentiel de production d'huile de pépins de raisin, très supérieur à leurs besoins. Mais les difficultés de process des pépins de raisin et les volumes concernés ne présentent à leurs yeux qu'un intérêt limité, d'autant qu'ils disposent d'une filière d'élimination des pépins produits.

\subsection{Codex Alimentarius et spécifications FEDIOL}

Le différentiel de prix de l'huile de pépins de raisin par rapport aux huiles de gros volumes telles que l'huile de tournesol pouvant atteindre 200 à $250 \%$, certains acteurs ont pu être tentés de couper des huiles ayant pour appellation «huile $100 \%$ pépins de raisin », d'autant que les normes du Codex Alimentarius s'avèrent généreusement larges. Elles imposent aux huiles de pépins de raisin, en sus des caractéristiques qualitatives communes à toutes les huiles, des fourchettes larges de teneurs en certains acides gras et une teneur en érythrodiol supérieure à $2 \%$ des stérols totaux $(\mathrm{FAO} /$ WHO, 2001), cf. Tableau 2. 
D'où la volonté des quatre gros acteurs européens du marché de l'huile de pépins de raisins (deux acteurs espagnols, un acteur français et un italien) de se fédérer et de proposer une spécification (voir Tab. 2), discutée et approuvée au sein de l'association européenne des huiliers FEDIOL, basée notamment sur la teneur en oméga- 6 (fourchette $65 / 78 \%$ au lieu de $58 / 78 \%$ ) et surtout sur la teneur en acide béhénique, un acide gras saturé de formule chimique $\mathrm{CH} 3-(\mathrm{CH} 2) 20-\mathrm{COOH}$ (maximum de $0,2 \%$ au lieu de $0,5 \%$ ) qui permet de tracer toute adultération du produit par coupage à l'huile de tournesol. Les spécifications développées par FEDIOL, en coopération avec ses membres, sont des recommandations non contraignantes élaborées pour l'utilisation dans les contrats entre membres et non-membres (Tab. 3).

\section{Conclusion}

L'huile de pépins de raisin rentre bien dans la définition d'un marché de niche, en raison des très faibles volumes produits. Elle incarne également la réussite d'une démarche de valorisation, qui a permis à un coproduit des marcs de vinification d'être transformé en un produit noble, largement valorisé. Pour autant, l'huile de pépins de raisins demeure un produit jeune, avec un marché qui a besoin de se réglementer, afin d'éviter que des fraudes (huiles coupées) n'en ternissent l'image.

Remerciements. Les sources chiffrées du marché de l'huile de pépins de raisin proviennent du seul et actuel acteur français, la Société Provence Huiles qui commercialise son huile dans le monde entier.

\section{Références}

Anses. 2016. Tables de composition nutritionnelle Ciqual. Disponible sur: https://pro.anses.fr/tableciqual/index.htm. Last consult : 2017/05/23.

FAO/WHO. 2001. The Codex Alimentarius Second edition (Revised 2001). Vol. 8. Section 2. Codex Standards for Fats and Oils from Vegetable Sources. Codex Standard for Named Vegetable Oils (CODEX-STAN 210-1999). Available from: http://www.fao. org/docrep/004/y2774e/y2774e04.htm\#bm4.2. Last consult : $2017 / 05 / 23$.

FEDIOL. 2016. Specifications for grapeseed oil. Available from: http://www.fediol.be/data/16SPEC212\%20FEDIOL\%20specifi cations $\% 20$ for $\% 20$ grapeseed $\% 20$ oil $\% 20$ FINAL.pdf. Last consult : $2017 / 05 / 23$.

Citation de l'article : Pierron J-C. 2017. L'huile de pépins de raisin en France et dans le monde. OCL 24(5): D502. 